\title{
Effects of Changes in Motor Unit Size on Transmitter Release at the Frog Neuromuscular Junction'
}

\author{
ALBERT A. HERRERA*,2 AND ALAN D. GRINNELL‡ \\ *Neurobiology Section, Department of Biological Sciences, and Program in Neural, Informational, and Behavioral Sciences, University of \\ Southern California, Los Angeles, California 90089 and $\ddagger$ Department of Physiology and Jerry Lewis Neuromuscular Research Center, \\ University of California, Los Angeles, California 90024
}

\begin{abstract}
The dependence of transmitter release and synaptic effectiveness on the size of a neuron's peripheral field was studied using neuromuscular junctions in sartorius muscles of adult frogs (Rana pipiens). The size of the peripheral field (motor unit size) was reduced by crushing the sartorius nerve and surgically removing half of the muscle fibers. Synapses thus formed were compared with those formed when crushed nerves reinnervated intact whole muscles, as well as with synapses in normal unoperated muscles. Indirect observations suggested that all motor axons participated in reinnervation and that motor unit size was indeed smaller in halfmuscles. Synaptic safety margins, as measured by the sensitivity of nerve stimulus-evoked twitching to low $\mathrm{Ca}^{2+}$, were substantially higher in muscles with reduced motor units. These higher safety margins were due to enhanced evoked transmitter release. In Ringer solution containing $\mathrm{Mg}^{2+}$ and lowered $\mathrm{Ca}^{2+}$, total evoked release and evoked release per unit nerve terminal length were approximately 2 -fold higher in muscles with reduced motor units, when studied 7 to 18 weeks postoperatively. A similar difference was seen when unblocked release was measured in a normal physiological solution, after blocking excitation-contraction coupling and muscle fiber action potential generation with formamide. Miniature endplate potential frequency in half-muscles was 2 to 3 times higher than in controls when tested in normal physiological solution, but was not significantly different in low $\mathrm{Ca}^{2+}, \mathrm{Mg}^{2+}$-containing solution. By 34 weeks postoperatively, there was no longer a difference in evoked release, even though the difference in motor unit size persisted. We conclude that synaptic effectiveness is inversely proportional to experimentally altered motor unit size, and that motoneurons in adult frogs retain the capacity to adjust transmitter output to peripheral field size.
\end{abstract}

It is increasingly apparent that many interesting forms of synaptic plasticity occur at the neuromuscular junction, where they can be easily studied. Long-term changes in the function of this synapse have been associated with aging, injury, disease, altered use, and

Received October 16, 1984; Revised December 10, 1984; Accepted December 11, 1984

\footnotetext{
${ }^{1}$ We are grateful to Frances Knight, David Scott, and Lisa Banner for expert technical help. This work was supported by United States Public Health Service Grants NSO6232 to A. D. G. and NS18186 to A. A. H. and by the Muscular Dystrophy Association.

${ }^{2}$ To whom correspondence should be addressed.
}

hibernation (reviewed in Grinnell and Herrera, 1981). Of particular interest are findings that the function of a motoneuron depends on the size of its peripheral innervation field, i.e., motor unit size. Within individual muscles of normal adult frogs, there is a direct correlation between motor unit size and synaptic effectiveness (Grinnell and Herrera, 1980a; Grinnell and Trussell, 1983; Trussell and Grinnell. 1985), although this relationship may not hold when motor units in different muscles are compared (Grinnell and Herrera, 1980b). Motoneurons that have sprouted to enlarge their fields appear to form less effective synapses (Bennett and Raftos, 1977; Bennett et al., 1979; Haimann et al., 1981; Slack and Hopkins, 1982) and also seem to be at a disadvantage when competing for synaptic connections with motoneurons supporting fewer synapses than normal (Brown et al., 1976; Brown and Ironton, 1978; Thompson, 1978; Wigston, 1980; Haimann et al., 1981). On the other hand, transmitter release from motor nerve terminals is increased when motor unit size is decreased by causing the nerve to reinnervate fewer muscle fibers than normal (Herrera and Grinnell, 1980; Ding, 1982b) or by crushing intramuscular nerves (Pockett and Slack, 1982).

Thus, during normal development, there seem to be mechanisms that adjust the level of transmitter output, and perhaps also competitive prowess, so that they are appropriate for a neuron's peripheral connections. It is important to further define these mechanisms and to ask whether they persist into adulthood, where they may deter mine much of a motoneuron's responses to changes in its environment. The present study examines these questions using frog neuromuscular junctions where synaptic effectiveness has been enhanced by decreasing motor unit size (Herrera and Grinnell, 1980). We find that this enhancement of transmitter release occurs in normal physiological solutions and that, at long postoperative times, the enhanced release from terminals in reduced motor units returns to normal levels.

\section{Materials and Methods}

In 54 adult Rana pipiens (body length, 4.5 to $6.5 \mathrm{~cm}$; anesthetized by immersion in $0.2 \%$ aqueous tricaine methanesulfonate), the left sartorius nerve was crushed near its entrance to the muscle. The sartorius muscle itself was bisected with a longitudinal cut, and muscle fibers in the lateral half of the muscle were discarded. As has been described previously (Dennis and Miledi, 1974a; DeCino, 1981; Ding, 1982a), the first reinnervated endplates were detected about 7 days after the nerve crush, and reinnervation was essentially complete by about 30 days. Control operations where the nerve was crushed but the muscle left intact were performed on 59 frogs obtained from the same supplier at the same time. Unless otherwise noted, acute experiments were performed on the reinnervated muscles 52 to 126 days after the operations, although some were kept for as long as 517 days Sartorius muscles from 42 unoperated frogs were also studied.

After dissection, muscles were placed in a $5-\mathrm{ml}$ bath and superfused at 2 $\mathrm{m} / \mathrm{min}$ with normal frog Ringer solution (NFR) containing $116 \mathrm{mM} \mathrm{NaCl}, 2$ $\mathrm{mM} \mathrm{KCl}, 1.8 \mathrm{mM} \mathrm{CaCl}_{2} 1 \mathrm{mM} \mathrm{MgCl}$ and $1 \mathrm{mM} \mathrm{NaHCO}_{3}(\mathrm{pH} 7.2)$. In some experiments, $\mathrm{Ca}^{2+}$ was reduced or $\mathrm{Mg}^{2+}$ was omitted as noted. Bath 
temperature was maintained at $15^{\circ} \mathrm{C}$. Brief $(0.1 \mathrm{msec})$ stimuli were delivered to the nerve at 0.5 or $1 \mathrm{~Hz}$ with suction electrodes.

The average safety margin for neuromuscular transmission was estimated by recording isometric twitch tension evoked by nerve stimulation while lowering $\mathrm{Ca}^{2+}$ in the Ringer solution. As $\mathrm{Ca}^{2+}$ is lowered, total twitch tension falls as individual fibers become blocked. A given fiber will cease twitching when transmission at the strongest of its two to four widely separated junctions becomes subthreshold. Thus, at the particular low $\mathrm{Ca}^{2+}$ concentrations used $(0.6$ and $1.0 \mathrm{~mm})$, the amount of twitch tension remaining relative to the amount of tension generated in normal Ringer solution $\left(1.8 \mathrm{mM} \mathrm{Ca}^{2+}\right)$ is a measure of safety margins at junctions in that muscle. In previous studies, such differences in safety margin were shown to be due primarily to presynaptic factors and were well correlated with average differences in transmitter release (Grinnell and Herrera,1980b; Herrera and Grinnell, 1980, 1981). Tension was measured with a Statham UC2 force transducer (maximal sensitivity, $1.1 \mathrm{mV} /$ dyne). Because transmitter release is affected by muscle stretch (Turkanis, 1973), all muscles were carefuly adjusted to 1.05 times rest length for these measurements of safety margin.

For intracellular recording, muscles were stretched to 1.1 times rest length and muscle fibers were impaled with one or two glass microelectrodes filled with $3 \mathrm{M} \mathrm{KCl}$. During recording, endplates were viewed through a compound microscope (Zeiss $40 / 0.75$ water immersion objective; $\times 400$ magnification) modified for Hoffman modulation contrast. All microelectrode penetrations were made within $50 \mu \mathrm{m}$ of the endplate, many of which could be seen well with these optics. Potentials were measured and analyzed by computer. Quantal content was estimated under three conditions:

1. In Ringer solution containing $0.3 \mathrm{mM} \mathrm{Ca}^{2+}$ and $1 \mathrm{mM} \mathrm{Mg}^{2+}$. For quantal analysis under these conditions, samples of at least 50 miniature endplate potentials (MEPPs) and 128 endplate potentials (EPPs) evoked at $1 \mathrm{~Hz}$ were obtained. Quantal content $(m)$ was calculated by the direct method $(m=$ mean EPP/mean MEPP), which was in good agreement with the method of failures; i.e., $m=\ln$ (number of trials)/(number of failures), at low quantal contents. Nonlinear summation correction was performed with the method of Martin (1955), assuming a reversal potential of $-15 \mathrm{mV}$.

2. In NFR without $\mathrm{Mg}^{2+}$, after pretreatment with $2 \mathrm{M}$ formamide in NFR to block excitation-contraction coupling (Escalona de Motta et al., 1982a; Escalona de Motta et al, 1982b). This was done in an attempt to use the direct method of calculating quantal content under conditions in which transmitter release was unblocked, but without using curare and the coefficient of variation method (see below). The methods used for formamide treatment and recording have been described previously (Herrera, 1984) Since formamide may have presynaptic effects, we were very careful to treat experimental and control muscles identically. Endplate potentials were corrected for nonlinear summation by the method of McLachlan and Martin (1981). Quantal content was calculated with the direct method.

3. In NFR containing $1 \mathrm{mM} \mathrm{Mg}^{2+}$ and to 2 to $6 \mu \mathrm{M}$ d-tubocurarine chloride. It was of interest to see how results obtained with the coefficient of variation $(\mathrm{CV})$ method in curare-treated muscles compared with those obtained using the direct method in formamide-treated muscles, even though the CV method is inherently less accurate (see "Discussion"). EPPs were corrected for nonlinear summation using Martin's (1955) correction. Depression of EPPS in a train was found to be quite pronounced even with stimulus frequencies as low as $0.05 \mathrm{~Hz}$. The contribution of depression to overall EPP variation was reduced by calculating quantal content (as $m=1 / \mathrm{CV}^{2}$ ) separately for small groups of successive EPPs (usually 5 to 10 ) and then averaging the separate results.

Intracellular recording was also used to estimate the incidence of focal polyneuronal innervation, the convergence of two or more synaptic inputs onto the same postsynaptic site. Muscles were blocked with the minimal concentration of curare necessary to block twitching (2 to $6 \mu \mathrm{M})$, and efforts were made to subdivide the EPP by varying the amplitude, polarity, and duration of a stimulus applied to the nerve

Certain aspects of the structure and function of frog neuromuscular junctions such as sprouting (Wernig et al., 1980a, b) and polyneuronal innervation (Herrera, 1984) are seasonally variable in freshly collected animals (reviewed in Grinnell and Herrera, 1981). There is no information on how these seasonal patterns are affected by laboratory maintenance for long periods of time under constant conditions, as was done in the present study. To insure that seasonal variation did not influence the current results, data were acquired at all seasons over a 5-year period, with experimental and control muscles studied simultaneously. Throughout the study, we used similarly sized frogs that were obtained from a supplier at the same time and maintained identically.

In most cases, electrophysiological studies were done on identified endplates. An additional micropipette, beveled and filled with $4 \%$ aqueous
Chicago Blue 6B, was used to mark the muscle fiber immediately adjacent to the endplate being studied by pressure injection of a small bolus of dye. After each experiment, muscles were stained with a combination of nitroblue tetrazolium (NBT) nerve terminal stain (Letinsky and DeCino, 1980) and cholinesterase (ChE) stain (Karnovsky, 1964). Marked endplates were relocated and drawn with a light microscope drawing tube. A computer and digitizing tablet were used to measure the total length of nerve terminal lying within synaptic gutters outlined by ChE reaction product. This length was taken as an approximation of the amount of nerve terminal that was actively secreting transmitter. Results were expressed as number of transmitter quanta released per $100-\mu \mathrm{m}$ nerve terminal length per nerve impulse.

Data samples which were not normally distributed are described by their median and interquartile range (IQR) and compared with the Mann-Whitney $U$ test. Normally distributed data are described by their mean and standard error of the mean and compared with standard parametric statistics.

\section{Results}

Before assessing synaptic effectiveness, the following observations were made at all postoperative times in order to compare motor unit sizes in experimental and control muscles. (1) Histological examination revealed that NBT-stained nerve terminal processes refilled the old ChE-outlined synaptic gutters to the same extent in experimental ( $94 \pm 9 \%$ (SE); $n=102$ ) and control ( $94 \pm 7 \% ; n=$ 206) muscles. Reinnervation appeared to be restricted to these old endplate sites, since ectopic junctions, which are easily recognized for many months after formation (Ding, 1982b), were not seen. (2) As judged by the number of increments in twitch tension produced by increasing or decreasing the strength of a stimulus applied to the whole nerve, there was no significant difference $(p<0.05)$ in the number of motor axons innervating experimental $(9.9 \pm 0.5 ; n=$ 14), control $(9.6 \pm 0.9 ; n=9)$, and normal $(8.5 \pm 0.4 ; n=14)$ muscles. (3) Maximal twitch tension in experimental muscles (106 $\pm 11 \mathrm{mN} ; n=16$ ) was substantially less than in control muscles $(147 \pm 20 \mathrm{mN} ; n=13)$, and very little muscle regeneration was observed. (4) Motor unit size was approximated by measuring the amplitude of the twitch increments seen when individual motor axons were differentially stimulated by grading the stimulus to the whole nerve and is expressed here as a percentage of the twitch tension produced when all axons are stimulated. After such normalization, motor unit size was not significantly different in experimental $(10.1$ $\pm 0.7 \% ; n=148)$, control $(10.5 \pm 0.8 \% ; n=64)$, and normal $(12.2$ $\pm 1.0 \% ; n=107)$ muscles. Taken together, these observations suggest that all motor axons regenerated and had a more or less equal chance of innervating each endplate, resulting in smaller motor units in the experimental case. This conclusion was further supported by measurements of the incidence of focal polyneuronal innervation which, at early postoperative times, was significantly less in experimental muscles (19\%, 10 muscles) compared with control muscles $(40 \%, 10$ muscles; $p<0.002)$. This interesting difference in polyneuronal innervation as a function of motor unit size and its fate at longer postoperative times is currently under investigation (A. A. Herrera and M. W. Werle, unpublished data).

Differences in synaptic effectiveness could be assessed most rapidly by measuring the tension of twitches evoked by single nerve stimuli while varying $\mathrm{Ca}^{2+}$ in the bathing Ringer solution. The extent to which the tension falls in any low $\mathrm{Ca}^{2+}$ is thus an average measure of safety margins at junctions in that muscle. Figure 1 shows that, at early postoperative times ( 7 to 18 weeks), when $\mathrm{Ca}^{2+}$ was lowered to $1 \mathrm{mM}$, twitch tension in reinnervated half sartorius muscles $(E)$ only fell to a mean of $77 \pm 4 \%$ of the tension in normal Ringer solution containing $1.8 \mathrm{~mm} \mathrm{Ca}^{2+}$. This was significantly more than the tension remaining in reinnervated whole sartorius muscles $(C$, $52 \pm 5 \% ; p<0.001$ by $t$ test) or normal unoperated muscles $(N$, $42 \pm 4 \% ; p<0.001$ ). Similarly, in $0.6 \mathrm{mM} \mathrm{Ca}^{2+}$, mean residual twitch tension in half sartorius muscles $(24 \pm 4 \%$ ) was significantly higher than in whole sartorius muscles $(3.6 \pm 1.0 \% ; p<0.01)$ or normal muscles $(4.4 \pm 1.9 \% ; p<0.001)$.

Synaptic effectiveness was also assessed by measuring transmitter release as EPP quantal content under several different exper- 

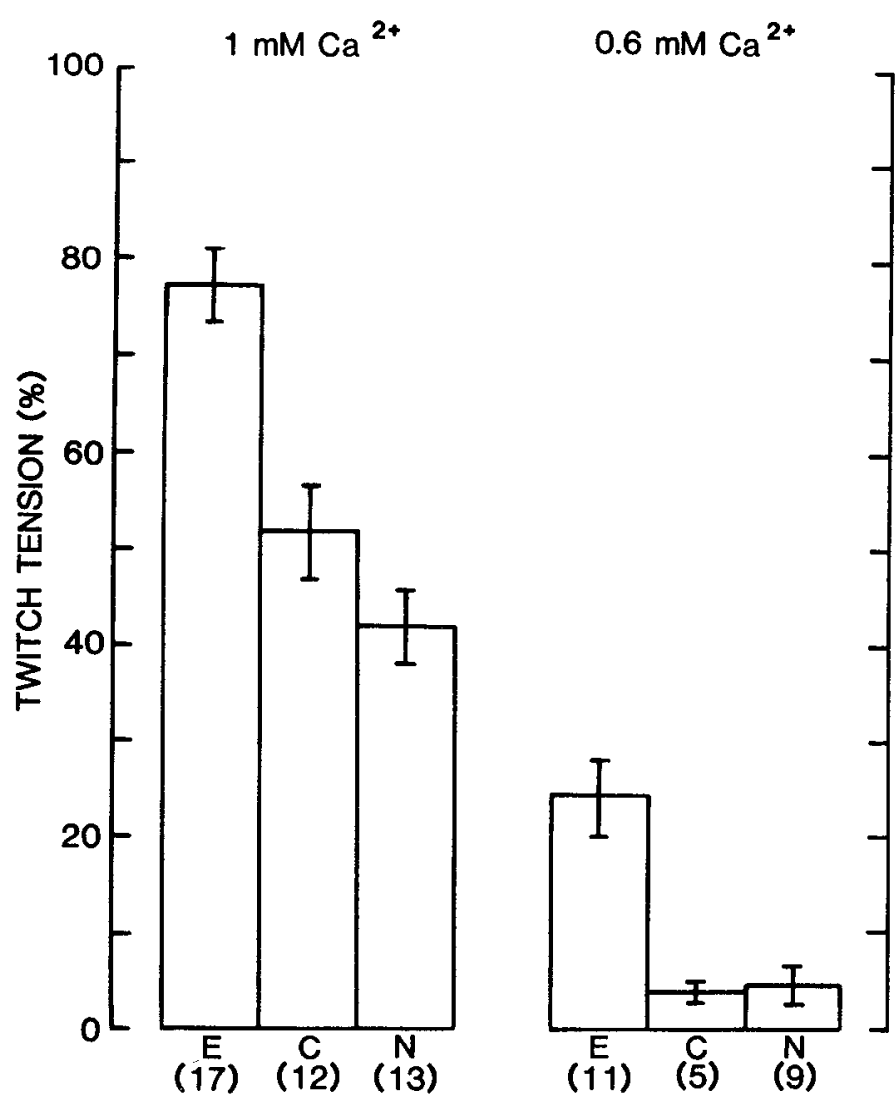

Figure 1. Synaptic safety margins in reinnervated half sartorius muscles (experimental, $E$ ), reinnervated whole sartorius muscles (control, $C$ ), and unoperated sartorius muscles (normal, $N$ ) examined 7 to 18 weeks postoperatively. Safety margin is expressed as the mean nerve stimulus-evoked twitch tension remaining after $\mathrm{Ca}^{2+}$ is lowered to 1.0 or $0.6 \mathrm{~mm}$, relative to tension generated in $1.8 \mathrm{~mm}$. Bars, SE. Number of observations is in parentheses

imental conditions. Quantal content measurements using the direct method in Ringer solution containing $0.3 \mathrm{mM} \mathrm{Ca}^{2+}$ and $1 \mathrm{mM} \mathrm{Mg}^{2+}$ are shown in Figure 2. In the postoperative time range of 7 to 18 weeks, median release from terminals in muscles with reduced motor unit size (4.6) was twice as great as release from control muscles with larger molor unils $(2.2 ; p<0.05$ by Mann-Whitney $U$ test). Mean values differ similarly, and parametric statistics indicate even greater significance $(p<0.0001)$. At postoperative times greater than 34 weeks, however, there is no longer a significant difference between median release from reinnervated half sartorius terminals (1.2) and that from reinnervated whole sartorius terminals (1.8; $p>0.05)$, even though the difference in motor unit size persists. This is not due to a change in release from reinnervated whole sartorius terminals (early median, 2.2; late, 1.8), but rather to the fact that the enhanced release brought about by changing motor unit size does not persist. Release from half sartorius terminals is significantly lower at late times (median 1.2) compared with early times (median, $4.6 ; p<0.002$ by $U$ test).

Quantal content in $0.3 \mathrm{mM} \mathrm{Ca}^{2+}$ and $1 \mathrm{~mm} \mathrm{Mg}^{2+}$ Ringer solution was also measured at endplates that were marked during recording and relocated after histological staining. Transmitter release was then normalized to nerve terminal length. The median number of quanta released per $100-\mu \mathrm{m}$ terminal length in small motor units (1.33; IQR $=0.87-1.89$ ) was significantly higher than in large motor units $(0.52, \mathrm{IQR}=0.025-1.09 ; p<0.002$ by $U$ test $)$.

Although there were clear differences in stimulus-evoked transmitter release in $0.3 \mathrm{mM} \mathrm{Ca}^{2+} / 1 \mathrm{mM} \mathrm{Mg}^{2+}$ Ringer solution, similar differences in spontaneous transmitter release were not found. At 7 to 18 weeks postoperatively, there were no significant differences
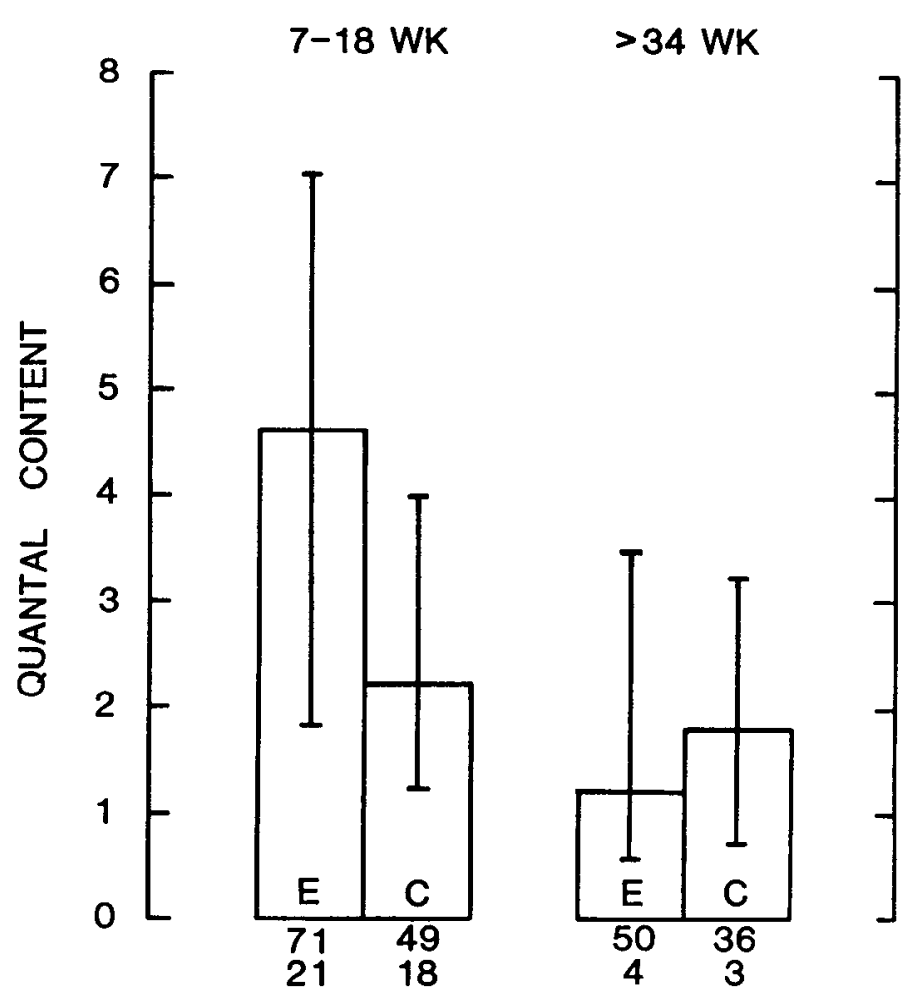

Figure 2. Quantal content in Ringer solution containing $0.3 \mathrm{mM} \mathrm{Ca}^{2+}$ and $1 \mathrm{mM} \mathrm{Mg}^{2+}$ for endplates in reinnervated half sartorius muscles $(E)$ and reinnervated whole sartorius muscles $(C)$ at two different ranges of postoperative times. Rectangles show medians, and vertical lines show interquartile ranges. Below the graph are the number of endplates (top) and number of muscles (bottom) sampled.

$(\rho>0.05)$ in MEPP frequency between reinnervated half muscles $(0.33 \pm 0.05 \mathrm{H} 7 ; n=53)$, reinnervated whole muscles $(0.33 \pm 0.04$ $\mathrm{Hz} ; n=49)$, and normal unoperated muscles $(0.29 \pm 0.03 \mathrm{~Hz} ; n=$ 105). Similar results were obtained from long-term operated animals (>34 weeks). At these long times, MEPP frequency in half muscles $(0.26 \pm 0.04 \mathrm{~Hz} ; n=43)$ did not differ significantly from that in whole muscles $(0.35 \pm 0.05 \mathrm{~Hz} ; n=28)$, nor did either of these differ significantly from MEPP frequency in the same type of muscle examined at early times or in normal unoperated muscles.

While calculations of EPP quarital content and MEPP frequency made in solutions of low $\mathrm{Ca}^{2+}$ may be more accurate, measurements made under conditions in which release is unblocked are more physiologically relevant and may be more legitimate if regenerated terminals differ in their sensitivity to divalent cations. We measured unblocked release by pretreating muscles with $2 \mathrm{M}$ formamide to block excitation-contraction coupling and calculating quantal content using the direct method; i.e., $m=$ mean EPP/mean MEPP. Possible presynaptic effects of formamide introduce uncertainty into this approach, but we controlled for this by treating experimental and control muscles identically. Figure 3 shows the results. At 8 to 16 weeks postoperatively, median quantal content at endplates in muscles with reduced motor units (77.3) was significantly higher than quantal content at endplates in muscles with larger motor units (43.7; $p<0.001$ by $U$ test). Thus, stimulus-evoked transmitter release is higher in experimental muscles, even at high levels of release. This difference was not apparent in measurements of quantal content using the CV method in curare-treated muscles. Mean calculated quantal content at junctions in half sartorius muscles $(324 \pm 61 ; n=12)$ was not significantly different than quantal content in whole sartorius muscles $(477 \pm 84 ; n-17 ; p>0.20$ by $t$ test). The higher release from whole sartorius terminals may be partially explained by the fact that in these small samples, terminal length was $12 \%$ higher for the whole sartorius (mean terminal length 

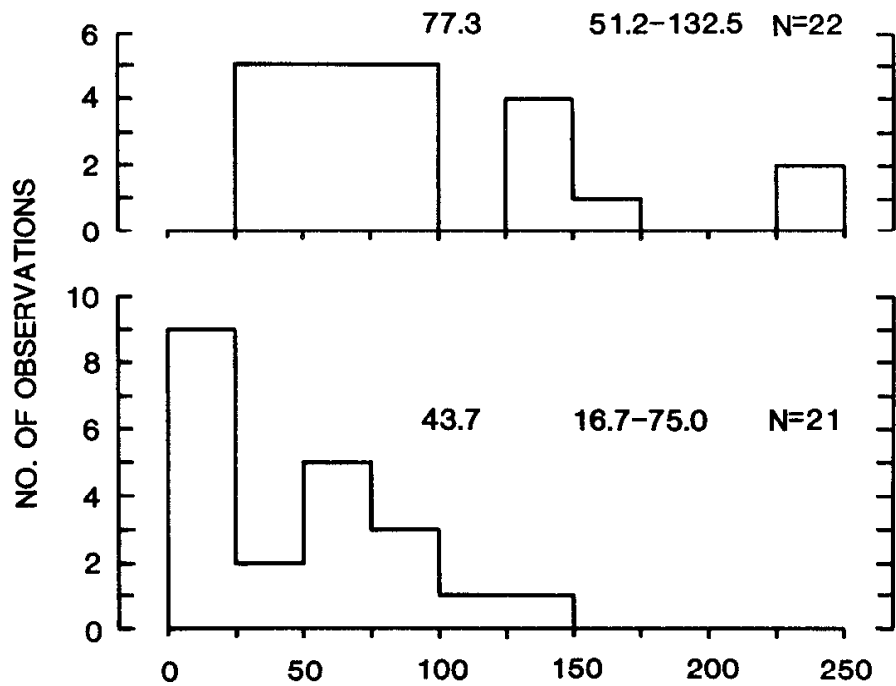

Figure 3. Endplate potential quantal content in normal Ringer solution after formamide treatment for reinnervated half sartorius muscles (top) and reinnervated whole sartorius muscles (bottom) examined 8 to 16 weeks postoperatively. Numbers show medians, interquartile ranges, and number of observations. Excitation contraction coupling and muscle fiber action potential generation blocked by formamide pretreatment.

$=543 \mu \mathrm{m}$ for endplates in whole sartorius muscles, $484 \mu \mathrm{m}$ for half sartorius endplates). The discrepancy between results obtained after formamide treatment and with curare is treated in the discussion. Transmitter release in normal Ringer solution at long postoperative times was not tested.

In contrast to results obtained with muscles bathed in a low $\mathrm{Ca}^{2+}$, $\mathrm{Mg}^{2+}$-containing solution, experimental muscles did show higher spontaneous release when tested in normal physiological solution. In NFR, mean MEPP frequency in reinnervated half sartorius muscles $(0.38 \pm 0.05 \mathrm{~Hz} ; n=44)$ was significantly higher than mean MEPP frequency in reinnervated whole muscles $(0.13 \pm 0.07 ; n=25 ; p<$ 0.0002 ). A similar relative difference was seen in a separate group of endplates tested in NFR after formamide treatment to block excitation-contraction coupling (mean for half sartorius, $0.27 \pm 0.05$ $\mathrm{Hz}, n=22$; whole sartorius, $0.13 \pm 0.04 \mathrm{~Hz}, n=21 ; p<0.05$ )

\section{Discussion}

In confirmation of previous studies (Herrera and Grinnell, 1980), these results show that reducing the motor unit size of regenerating frog sartorius motoneurons enhances synaptic efficacy. This was demonstrated in 2 ways. First, we found a marked increase in synaptic safety margin, as measured by the effects of lowered $\mathrm{Ca}^{2+}$ on the nerve stimulus-evoked twitch. Second, there was an increase in evoked transmitter release, whether expressed as total quanta released or as quanta released per unit nerve terminal length. These changes were quite pronounced in the postoperative time range of 7 to 18 weeks. They are not permanent, however. At postoperative times greater than 34 weeks, synaptic effectiveness had declined to control levels.

This decrease in effectiveness at long times does not appear to be due to a delayed expansion of motor units. There was no longterm increase in muscle fiber number, the number of endplate sites per fiber, or in nerve terminal length per endplate. Recent observations (A. A. Herrera and M. W. Werle, unpublished data) suggest that the incidence of focal polyneuronal innervation actually decreases at long times after reinnervation, as has been described by others (McArdle, 1975; Haimann et al., 1981).

Evoked transmitter release was measured by several different methods, each with inherent drawbacks. The most theoretically sound approach is the direct method, which is simply a statement of the quantal hypothesis; i.e., $m=$ mean EPP/mean MEPP. Unfortunately, muscle contraction movement restricts this approach to unphysiological conditions where release is substantially blocked by low $\mathrm{Ca}^{2+}$ or high $\mathrm{Mg}^{2+}$. Under such conditions, release from terminals in reduced motor units was approximately 2 -fold higher. To search for differences at normal high levels of release, we applied the direct method in muscles bathed in NFR after blocking excitation-contraction coupling and muscle fiber action potential generation with formaninide (Herrera, 1984). Results were sirnilar to those seen in low $\mathrm{Ca}^{2+}$, with median values of quantal content about 1.8 -fold higher in muscles with reduced motor units.

Quantal content measured with the direct method after formamide treatment was substantially lower than that measured with the $\mathrm{CV}$ method in curare-treated muscles. In addition, the $\mathrm{CV}$ results gave no evidence of enhanced release in reduced motor units. However, the direct method results are in reasonable agreement with previously published measurements (see Herrera, 1984). Furthermore the $\mathrm{CV}$ method, as it is usually applied $\left(m=1 / \mathrm{CV}^{2}\right)$, may be in serious error if the release process follows binomial rather than Poisson statistics (Miyamoto, 1975; Wernig, 1975). Possible presynaptic effects of curare (reviewed in Grinnell and Herrera, 1981) and the contribution of depression to total EPP variation represent additional sources of error. In fact, when the CV method was applied to EPPs recorded from formamide treated endplates, the resulting quantal content values agreed poorly with those obtained using the more reliable direct method. For junctions with high quantal content, the $\mathrm{CV}$ method frequently yielded results 2 to 3 times higher. This is expected since, at these junctions, release deviates most from a Poisson process. Because of these uncertainties, we consider the CV method to be inherently less accurate.

Release from terminals in reduced motor units was also significantly higher after normalizing to nerve terminal length. In other studies (Grinnell and Herrera, 1980b; Herrera and Grinnell, 1981; Pawson and Grinnell, 1984), release per unit terminal length proved to be a useful measure of the inherent transmitter-releasing ability of a nerve terminal. However, these previous studies were performed on intact, not regenerated endplates. One assumption in this approach is that release is relatively uniform along the length of terminal branches. There is evidence that, in many normal frog junctions, there is relatively less release from the distal portions of long terminal branches (D'Alonzo and Grinnell, 1985). In ultrastructural studies on similar reinnervated preparations (A. A. Herrera and A. D. Grinnell, unpublished data), we find that the width of synaptic contact between the nerve terminal and the muscle can be quite variable. Significant portions of regenerated nerve terminals occupying synaptic gutters and otherwise appearing normal in the light microscope are actually separated from the postsynaptic membrane by as much as $1 \mu \mathrm{m}$ and contain few or no vesicles. These observations suggest that release along the length of regenerated nerve terminal branches may be quite nonuniform, and there is some physiological evidence to support this view (Dennis and Miledi, 1974b). However, in other physiological studies, release from regenerated terminals was uniform (DeCino, 1981). Until the legitimacy of normalizing transmitter release to terminal length in reinnervated endplates is established by future physiological and morphological studies, such results should be viewed with caution.

The effects of altering motor unit size on the rate of spontaneous transmitter release appeared to depend on the solution bathing the muscle during testing. As would be expected for junctions having higher quantal content (Kuno et al., 1971; Grinnell and Herrera, $1980 \mathrm{~b})$, muscles with reduced motor unit sizes did show higher spontaneous release levels in normal Ringer solution, even after formamide pretreatment. However, in low $\mathrm{Ca}^{2+}, \mathrm{Mg}^{2+}$-containing Ringer solution, MEPP frequency in experimental and control muscles did not differ significantly at any time examined. Why a difference was not seen in this case was unclear. Several studies have shown that the return of spontaneous transmitter release lags behind the return of evoked release in regenerating frog motor nerve terminals (DeCino, 1981; Ding, 1982a), but this condition seems to persist only for the first 4 weeks following nerve crush. By 8 to 40 
weeks after nerve crush in the frog cutaneous pectoris, MEPP frequency in regenerated terminals is not different from normal (Ding, 1982a).

The simplest interpretation of the present results and those obtained earlier (Grinnell and Herrera, 1980a; Grinnell and Trussell, 1983; Trussell and Grinnell, 1985) is that there is some substance(s), manufactured in the soma and transported to the terminals, that is necessary for transmitter release. During normal development, the amount of this substance each terminal comes to receive is a function of motor unit size. lerminals in larger motor units each receive more and so, on the average, form more effective synapses. When motor unit size is experimentally reduced, each terminal receives a larger share of this substance, and release is enhanced. The ability to adjust production and/or transport of this substance to match peripheral field size apparently persists into adulthood, since the enhanced release from terminals in reduced motor units eventually returns to normal levels. This conclusion was supported by similar work on a related preparation (Pockett and Slack, 1983).

\section{References}

Bennett, M. R., and J. Raftos (1977) The formation and regression of synapses during re-innervation of axolotl striated muscles. J. Physiol. (Lond.) 265: 261-295.

Bennett, M. R., P. A. McGrath, and D. F. Davey (1979) The regression of synapses formed by a foreign nerve in a mature axolotl striated muscle. Brain Res. 173: 451-469.

Brown, M. C., and R. Ironton (1978) Sprouting and regression of neuromuscular synapses in partially denervated mammalian muscles. J. Physiol. (Lond.) 278: 325-348.

Brown, M. C., J. K. S. Jansen, and D. Van Essen (1976) Polyneuronal innervation of skeletal muscles in new-born rats and its elimination during maturation. J. Physiol. (Lond.) 261: 387-422.

D'Alonzo, A. J., and A. D. Grinnell (1985) Profiles of evoked release along the length of frog motor neve terminals. J. Physiol. (Lond.) 359: 235-258.

DeCino, P. (1981) Transmitter release properties along regenerated nerve processes at the frog neuromuscular junction. J. Neurosci. 1:308-317.

Dennis, M. J., and R. Miledi (1974a) Non-transmitting neuromuscular junctions during an early stage of end-plate reinnervation. J. Physiol. (Lond.) 239: 553-570.

Dennis, M. J., and R. Miledi (1974b) Characteristics of transmitter release at regenerating frog neuromuscular junctions. J. Physiol. (Lond.) 239: 571594.

Ding, R. (1982a) Lack of correlation between physiological and morphological features of regenerating frog neuromuscular junctions. Brain Res. 253: 47-55.

Ding, R. (1982b) Comparison of morphology and physiology of synapses formed at ectopic and original endplate sites in frog muscle. Brain Res. 253: $57-63$.

Escalona de Motta, G., Cordova, F., de Leon, M., and J. del Castillo (1982a) Inhibitory action of high formamide concentrations on excitation-contraction coupling in skeletal muscle. J.Neurosci. Res. 7: 163-178.

Escalona de Motta, G., D. S. Smith, M. Cayer, and J. del Castillo (1982b) Mcchanism of the excitation contraction uncoupling of frog skelctal muscle by formamide. Biol. Bull. 163: 276-286.

Grinnell, A. D., and A. A. Herrera (1980a) Synaptic efficacy depends on motor unit size in nomal Irog neurornuscular junclions. Soc. Neurosci. Abstr. 6: 571.

Grinnell, A. D., and A. A. Herrera (1980b) Physiological regulation of synaptic effectiveness at frog neuromuscular junctions. J. Physiol. (Lond.) 307: 301-317.
Grinnell A. D., and A. A. Herrera (1981) Specificity and plasticity of neuromuscular connections: Long-term regulation of motoneuron function. Prog. Neurobiol. 17: 203-282.

Grinnell A. D., and L. O. Trussell (1983) Synaptic strength as a function of motor unit size in the normal frog sartorius. J. Physiol. (Lond.) 338: 221241.

Haimann, C., A. Mallart, J. Tomas I Ferre, and N. F. Zilber-Gachelin (1981) Interaction between motor axons from two different nerves reinnervating the pectoral muscles of Xenopus laevis. J. Physiol. (Lond.) 310: 257-272.

Herrera, A. A. (1984) Polyneuronal innervation and quantal transmitter release in formamide-treated frog sartorius muscles. J. Physiol. (Lond.) 3b5: 26/280.

Herrera, A. A., and A. D. Grinnell (1980) Transmitter release from frog motor terminals depends on motor unit size. Nature 287: 649-651.

Herrera, A. A., and A. D. Grinnell (1981) Contralateral denervation causes enhanced transmitter release from frog motor nerve terminals. Nature 291: 495-497.

Karnovsky, M. J. (1964) The localization of cholinesterase activity in rat cardiac muscle by electron microscopy. J. Cell Biol. 23: 217-232.

Letinsky, M. S., and P. DeCino (1980) Histological staining of pre- and postsynaptic components of amphibian neurosmuscular junctions. J. Neurocytol. 9: 303-320.

Martin, A. R. (1955) A further study of the statistical composition of the endplate potential. J. Physiol. (Lond.) 130: 114-122.

McArdle, J. J. (1975) Complex end-plate potentials at the regenerating neuromuscular junction of the rat. Exp. Neurol. 49: 629-638.

McLachlan, E. M., and A. R. Martin (1981) Non-linear summation of endplate potentials in the frog and mouse. J. Physiol. (Lond.) 311: 307-324.

Miyamoto, M. D. (1975) Binomial analysis of quantal transmitter release at giycerol treated frog neuromuscular junctions. J. Physiol. (Lond.) 250: $121-142$.

Pawson, P. A. and A. D. Grinnell (1984) Posttetanic potentiation in strong and weak neurnmuscular junctions: Physiological differences caused by a differential $\mathrm{Ca}^{2+}$ influx. Brain Res. 323: 311-315.

Pockett, S., and J. R. Slack (1982) Pruning of axonal trees results in increased efficacy of surviving nerve terminals. Brain Res. 243: 350353.

Pockett, S., and J. R. Slack (1983) Ability of motoneurons to regulate quantal release and terminal growth after reduction in motor unit size. Brain Res. 258: 296-298

Slack, J. R., and W. G. Hopkins (1982) Neuromuscular transmission at terminals of sprouted mammalian motor neurons. Brain Res. 237: 121135.

Thompson, W. (1978) Reinnervation in partially denervated rat soleus muscle. Acta Physiol. Scand. 103: 81-91.

Trussell, L. O., and A. D. Grinnell (1985) The regulation of synaptic strength within motor units of the frog cutaneous pectoris muscle. J. Neurosci. 5 : 243-254.

Turkanis, S. A. (1973) Effects of muscle stretch on transmitter release at end-plates of rat diaphragm and frog sartorius muscle. J. Physiol. (Lond.) 230: $391-403$

Wernig, A. (1975) Estimates of statistical release parameters from crayfish and frog neuromuscular junctions. J. Physiol. (Lond.) 244: 207-221.

Wernig, A., M. Pecot-Dechavassine. and H. Stover (1980a) Sprouting and regression of the nerve at the frog neuromuscular junction in normal conditions and after prolonged paralysis with curare. J. Neurocytol. 9: 277-303.

Wernig, A., M. Pecot-Dechavassine, and H. Stover (1980b) Signs of nerve regression and sprouting in the frog neuromuscular synapse. In: Ontogenesis and Functional Mechanisms of Peripheral Synapses. INSERM Symposium No. 13. J. Taxi, ed., pp. 225-238, Elsevier/North-Holland Biomedical Press, Amsterdam.

Wigston, D. J. (1980) Suppression of sprouted synapses in axolotl muscle by transplanted foreign nerves. J. Physiol. (Lond.) 307: 355-366. 